\title{
Usability Chemical Application Based on User Experience Analysis
}

\author{
Alexander Setiawan ${ }^{1+}$, Silvia Rostaningsih ${ }^{2}$ \\ ${ }^{1,2}$ Informatics Department, Faculty of Industrial Technology - Petra Christian University
}

\begin{abstract}
An experience in making a usability chemical application is one of the results that will be produced by humans who interact with machine learning. Based on this experience contains various the things that can be learned, by namely designing the interface of the mobile application. From the results of this study, user experience is highly elaborated, especially for basic human needs in carrying out an activity in the use of mobile applications. In the usability factor, experience of this factor is very important in determining the effectiveness and efficiency of the system, technology, or environment around the user. The results of this study will produce a form of experience for human needs and the concept of usability and user convenience in mobile applications. The results of this interface chemistry study will benefit from the user experience in generating observations in chemistry in a graphical user interface that serves as a media interface and mobile application by completing a usability approach to user experience by testing applications to multiple cellular devices by utilizing the needs of these users.
\end{abstract}

Keywords: User Experience Analysis, Interface Chemical, Usability, Machine Learning.

\section{Introduction}

Technology is a necessity for everyone and is used to make it easier to complete or work on daily activities. The technology that is currently widely used is mobile technology. The mobile technology itself has developed quite rapidly so that it is needed by all users, so the development of mobile technology must be experience and impact for the user.

One mobile technology that is very interesting and that can be developed in this mobile application is a chemical application interface that uses an Augmented Reality model, this application is a technology that will combine objects in 2D or 3D into a three-dimensional real world and then project objects in real time. Based on these cases, the interface application will made chemical application based on User Experience Analysis. By evaluating with the User Experience approach it functions to find out something users feel, users feel happy, get ease, have that feeling of distress or feel satisfied when using a chemical interface.

\section{Literature Review}

\subsection{Usability and graphical user interface}

In life everyday, humans can interact with objects that are on surrounding it through the existence of this interface [1]. Interface is a bridge between the world of a product or system and user world [2]. That is, as well as the communication process general, the interface is a medium that connects communication between two domains (product / system and human) and play a role full in translate every action and from reaction both domains. Interface applies to several types or variants of products or systems.

In computer technology, one of the interfaces has associations and function as a medium between computational codes with a user application or software. In this case, the interface for the application has several types, one of which is a graphical interface (graphical interface). In a graphical interface, computational codes for specific functions represented through processed graphic elements such as through

\footnotetext{
+ Corresponding author. Tel.: + 6281-65411487; fax: +6231-8417658.

E-mail address: alexander@petra.ac.id; silvia@petra.ac.id.
} 
the icon. This is where the usability problem arises. Usability related to the effectiveness and efficiency of the interface and with the reaction users of the interface [2].

That usability measure exists as one's acceptance of a product or system based on an understanding and accuracy of a person's action / reaction to an interface. The responsibility of an interface design is huge here. A state-of-the-art system with an interface that is unable to represent the computing functions behind it and cannot meet the aspects that humans need when interacting with the interface, can cause a system to not be received by someone. Conversely, a simple system with an interface representative of computing functions and able to provide means which accommodates aspects that humans need when interacting with a system, it will be one of the reasons for receiving the product or the system by its users [3].

\subsection{User experience}

User Experience is part of Human Computer Interaction (HCI). The shift of usability to the scope of User Experience was caused by technological advances that caused almost all human activities not to be separated from technology. Where User Experience can understand feelings, motivations and value values more than a product, so users get more value than subjective efficiency, effectiveness and satisfaction [4]. User Interface Design is part of User Experience, but not all User Experience cases are related to User Interface. In general, User Interface focuses on the aesthetics of the appearance of a system while User Experience focuses on the convenience of users system. A User Experience designer does not design the same thing as a designer User Interface, User Experience designer creates a strategy that brings a solution [5]. So that a User Experience designer does not to have design with vector or graphics code programming in his work. Although different User Experience and User Interface have similarities which have the main purpose to increase user satisfaction in interacting with a system, product, or service [6]. The essence of UX is a user experience to find or get a value from a product, the product can used, useful, interesting, and can be used by users who have limitations [7].

\section{Analysis and Design System}

The design system there are 2 (two) parts, the overall menu design, search marker and view marker [8]. This process starts when a 3D object has appeared then the application detects whether there is more than one marker detected. If there are more than one marker, then it checks whether objects are in the database and has interactions with other objects. If there is an interaction then the animation will run, if not then the object will remain silent. The play animation process can be seen in Figure 1.

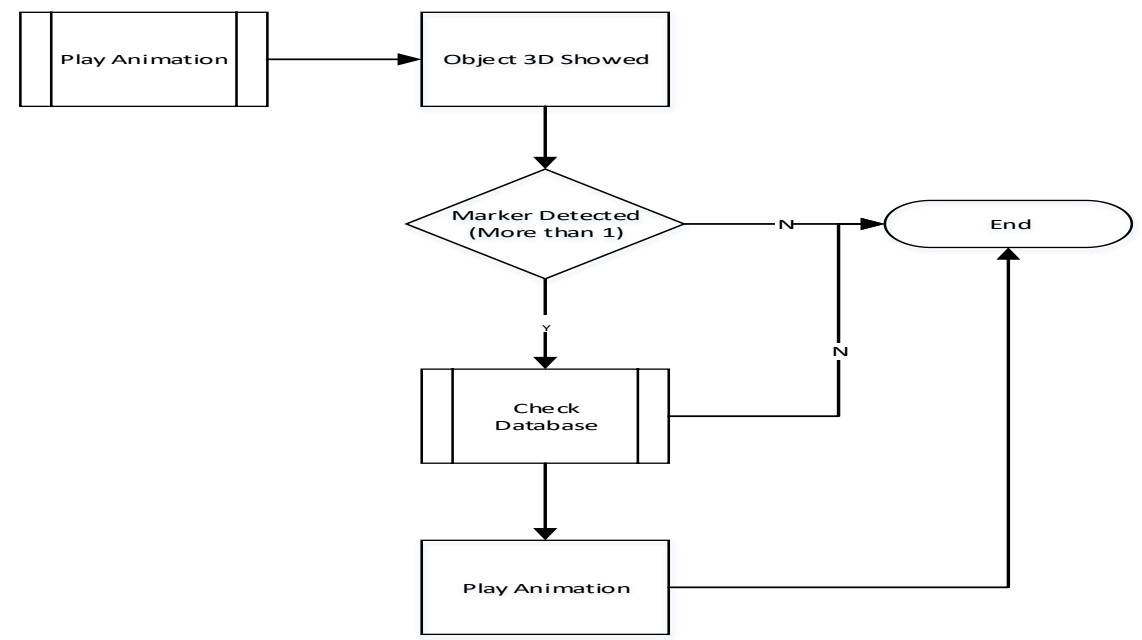

Fig. 1: Flowchart of Play Animation Process

The next process is to start checking whether objects are in the database. If an object is in a database, then checking again to see other objects detected are pairs of existing objects. The animation process will run if there is a match between objects detected with the contents of the database. The database check process can be seen in Figure 2. The next process is started when 3D objects have appeared on the screen mobile. Then check whether the user touches the screen with 2 fingers and both fingers are directed away or not. If 
the user touches the screen with 2 fingers and the two fingers are directed away, the 3D object will appear enlarged. The object zoom in process can be seen in Figure 3.

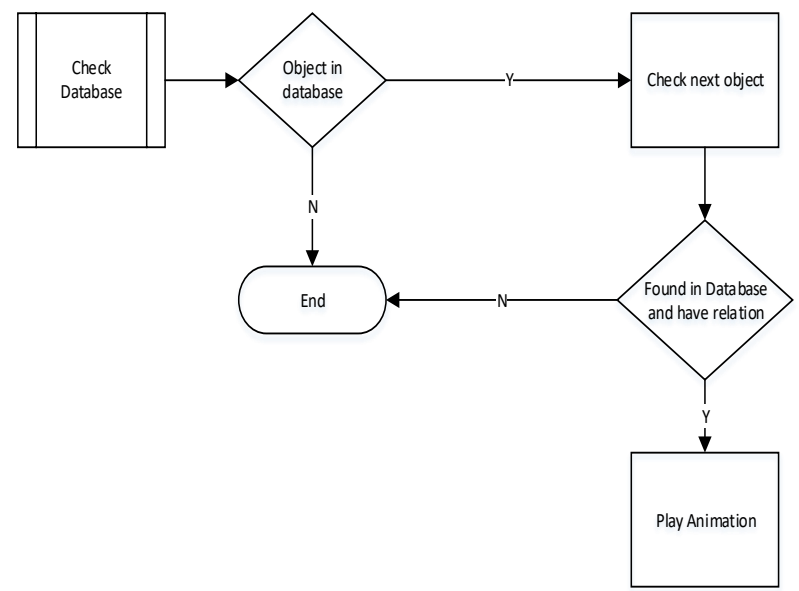

Fig. 2: Flowchart of Database Check Process

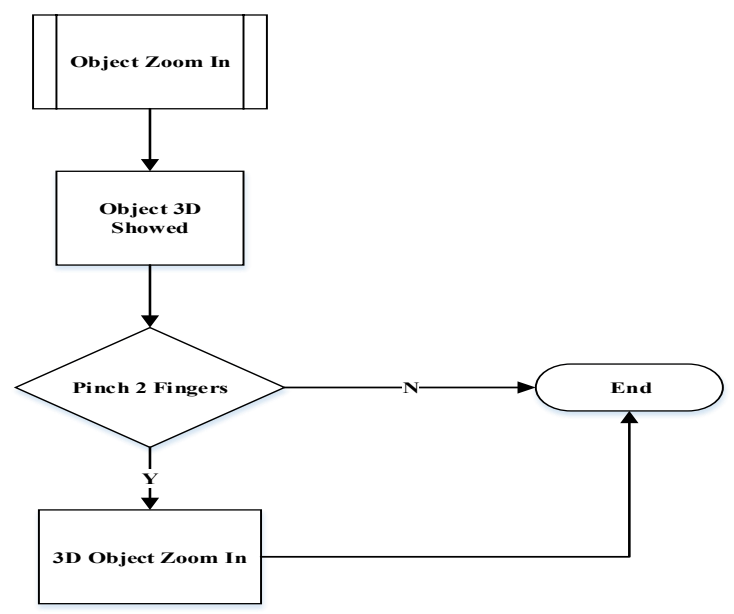

Fig. 3: Flowchart of Object Zoom Process

\section{Result and Discussion}

In the first step is to prepare each Marker along with a 3D model of its chemical elements. Each marker has a three-dimension collider component with a spherical shape, marked by thin green lines [9]. When sphere colliders come in contact with each other, it triggers an event. The collision is detected and included in the dataset. Here is a scene that contains every marker and chemical element in this program can be seen in Figure 4. Details of an AR Chemical object, the lowest layer is a marker, just above it is an overlay, along with 3D models can be seen in Figure 5.

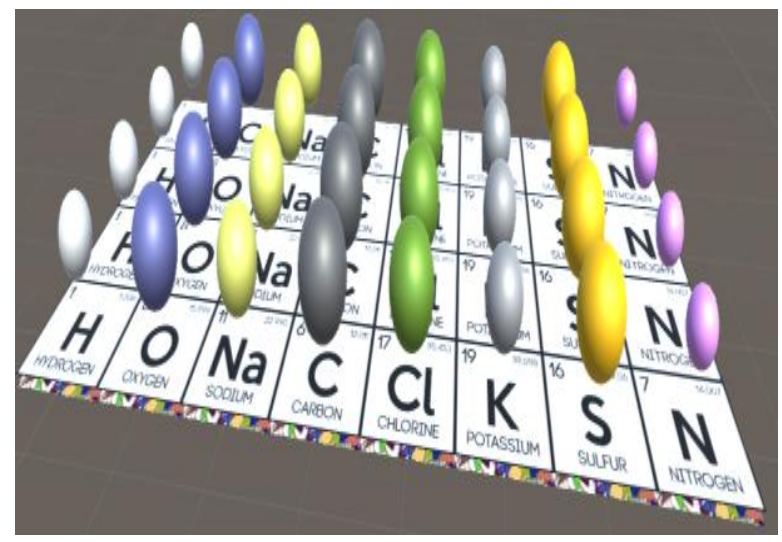

Fig. 4: Interface Object Chemical Element

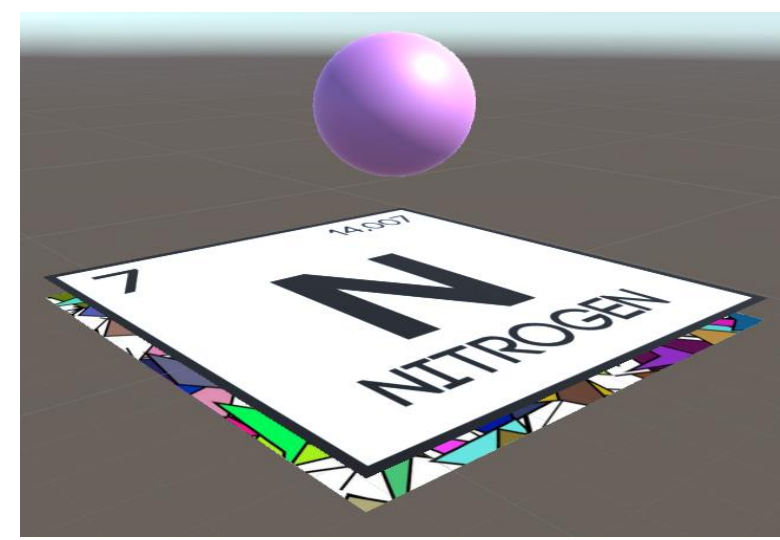

Fig. 5: The Result of Interface 3D Chemical Object

The testing android application devices using 2 devices, including Sony Xperia M2 and Lenovo A7-30. The specifications android devices android can be seen in Table 1.

Table 1: The Specifications Android Devices

\begin{tabular}{|c|c|c|c|}
\hline Device Type & $\begin{array}{c}\text { Operating } \\
\text { System }\end{array}$ & Display & CPU \\
\hline Sony Xperia M2 & Android 5.1.1 & $\begin{array}{c}4.8 "(960 \times 540 \text { pixels dan } \\
16,777,216-\text { colour TFT })\end{array}$ & $\begin{array}{c}1.2 \text { GHz Qualcomm } \\
\text { MSM8926 Quad Core }\end{array}$ \\
\hline Lenovo A7-30 A3300 & Android 4.2.2 & $\begin{array}{c}7.0 " \text { (600 x 1024 pixels dan } \\
16 M \text { colour TFT) }\end{array}$ & 1.3 GHz Quad Core \\
\hline
\end{tabular}

The Interface testing will be done by testing the Sony Xperia M2 mobile application on markers and then measured at a distance of how many markers can recognized and can bring 3D objects. This test was carried out by representing two markers, namely marker $\mathrm{H}$ and marker $\mathrm{C}$. The tests were carried out at a distance of $5 \mathrm{~cm}, 10 \mathrm{~cm}, 20 \mathrm{~cm}, 30 \mathrm{~cm}, 40 \mathrm{~cm}, 50 \mathrm{~cm}, 60 \mathrm{~cm}$, and $70 \mathrm{~cm}$. As for testing the interface on the Sony Xperia 
M2 mobile can be seen in Figure 6. For testing the interface on the Lenovo A7-30 mobile can be seen in Figure 7. The testing devices can be seen in Table 2 .

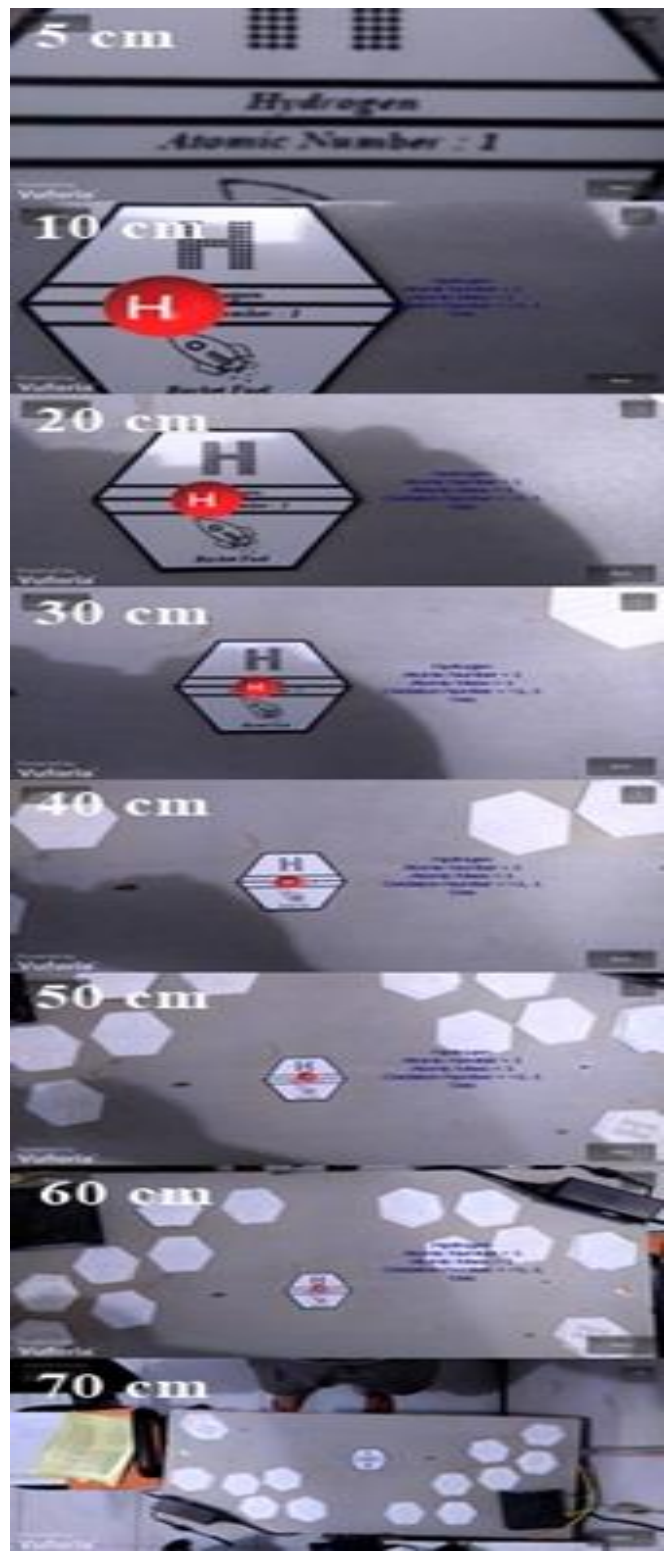

Fig. 6: The Interface Mobile Sony Xperia M2
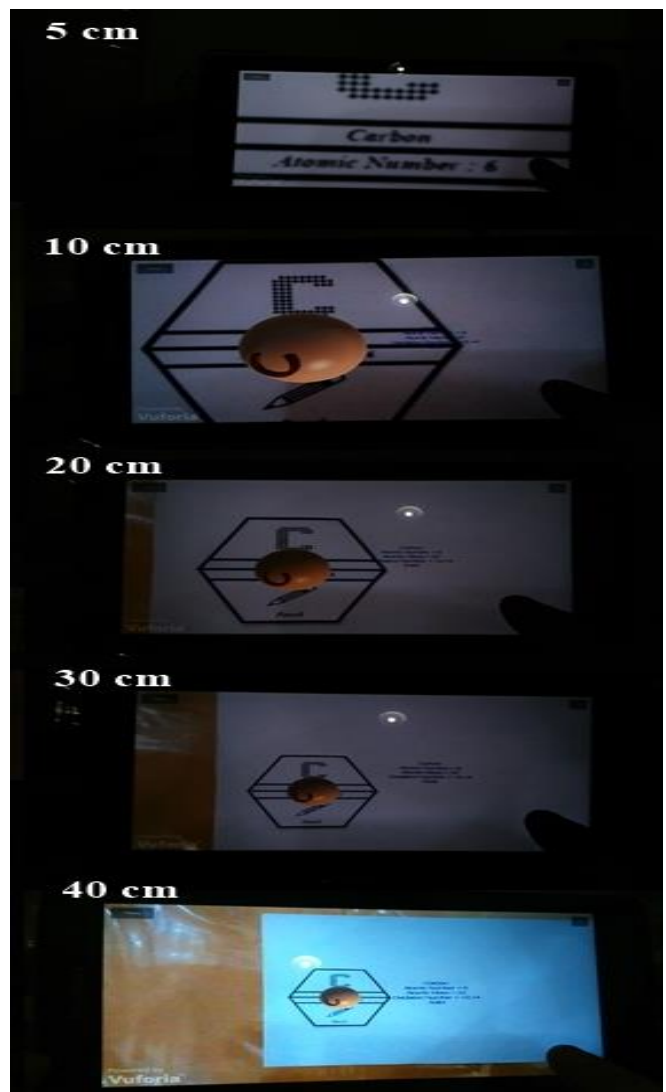

$50 \mathrm{~cm}$

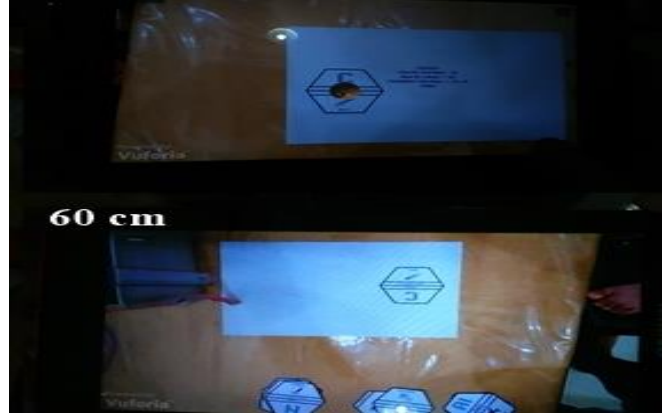

Fig. 7: The Interface Mobile Lenovo A7-30

Table 2: Testing Devices Marker H and Marker C

\begin{tabular}{|c|c|c|c|c|c|c|c|c|}
\hline \multicolumn{9}{|c|}{ Experiment with camera distance with marker $H$ and Marker $C$} \\
\hline \multirow[b]{2}{*}{ Device Type } & \multicolumn{8}{|c|}{ Distance } \\
\hline & $\begin{array}{c}5 \\
\mathbf{c m}\end{array}$ & $\begin{array}{c}10 \\
\mathrm{~cm}\end{array}$ & $\begin{array}{c}20 \\
\mathrm{~cm}\end{array}$ & $\begin{array}{l}30 \\
\mathrm{~cm}\end{array}$ & $\begin{array}{l}40 \\
\mathrm{~cm}\end{array}$ & $\begin{array}{c}50 \\
\mathrm{~cm}\end{array}$ & $\begin{array}{c}\mathbf{6 0} \\
\mathrm{cm}\end{array}$ & $\begin{array}{l}70 \\
\text { cm }\end{array}$ \\
\hline Sony Xperia M2 & - & $\mathrm{v}$ & $\mathrm{v}$ & $\mathrm{v}$ & $\mathrm{v}$ & $\mathrm{v}$ & $\mathrm{v}$ & - \\
\hline Lenovo A7-30 & - & $\mathrm{v}$ & $\mathrm{v}$ & $\mathrm{v}$ & $\mathrm{v}$ & $\mathrm{v}$ & $\mathrm{v}$ & - \\
\hline
\end{tabular}

This test has been done to find out how users assess the application of chemical interfaces. The test was carried out by dividing the questionnaire on several users with the target high school students who had tried the application. The following is the test results to the user about satisfaction of use, can be seen in Figure 8 . The Figure 8 shows that as many as $85.7 \%$ of respondents who agree to use this application are more interesting and easier to use. 


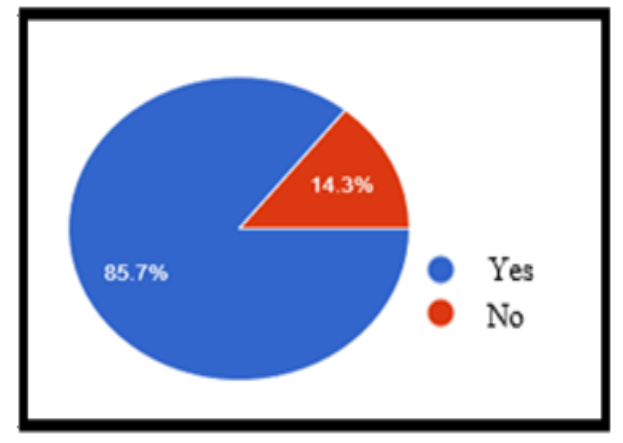

Fig. 8: The Result to The User About Usage Satisfaction

\section{Conclusion}

From the creation and design of the program can be concluded as follows:

- The Usability Chemical Applications can make the process is automatically integrated with the database form table element chemical.

- The minimum detection distance for markers is approximately $10 \mathrm{~cm}$, and the maximum distance of marker detection is approximately $50 \mathrm{~cm}$.

- Detection of markers by the camera mobile has been running well, markers can be recognized so as to be able to bring up a suitable object. The maximum number of markers that can detect and bring up objects is 8 pieces.

- According to the questionnaire, $85.7 \%$ of user rate the application are more interesting and easier to use.

\section{Acknowledgement}

This Research Project was funded by an Applied Product Research Grant, received in 2019, from Ministry of Research, Technology, and Higher Education of The Republic of Indonesia. We thank the Centre of Research Petra Christian University for the supports and guidance.

\section{References}

[1] Darmawan, Ruly. (2013). "Pengalaman, Usability, dan Antarmuka Grafis : Sebuah Penelusuran Teoritis." Journal of Visual Art and Design Vol 4. No. 2 : 95-102.

[2] Hackos, J.T. \& Redish, J.C. (1998). User and Task Analysis for Interface Design, John Wiley \& Sons, inc., Toronto.

[3] Coyne, R.D. (1998). Cyberspace and Heidegger's Pragmatics, Information Technology and People, Special Issue: Heidegger and Information Technology, 11(4), page.338-350.

[4] Towards the Integration of Transectorial IT Design and Evaluation. (2009). Retrieved October 21, 2017, from http://www.cost.eu/COST_Actions/ict/transectorial_it_design_and_evaluation

[5] Flowers, E. (2012). UX is not UI. Retrieved October 22, 2017, from http://www.helloerik.com/ux-is-not-ui

[6] Cline, A. (2015). The Difference Between UX and UI Design. Retrieved fromhttp://usabilitygeek.com/thedifference-between-uxand-ui-design/

[7] Lestari, N. P., Santosa I. P., Ferdiana, R., (2016). Prosiding Seminar Nasional Teknologi Informasi dan Komunikasi (SENTIKA 2016). Yogyakarta, 18-19 Maret 2016. ISSN : 2089-9815.

[8] Setiawan, A., Rostianingsih, S., \& Widodo, T. R. (2018). Application of Compound Bonding Based On Augmented Reality. In MATEC Web of Conferences (Vol. 248, p. 05007). EDP Sciences.

[9] S. Rostianingsih, A. Setiawan and C. I. Halim, (2018) "Ionic and Metallic Bonding Visualization Using Augmented Reality," 3rd Technology Innovation Management and Engineering Science International Conference (TIMES-iCON), Bangkok, Thailand, 2018, pp. 1-4. doi: 10.1109/TIMES-iCON.2018.8621665 